\title{
Melanoma-expressed CD70 is involved in invasion and metastasis
}

\author{
Christine Pich ${ }^{1,2,5}$, Guillaume Sarrabayrouse ${ }^{1,2,5}$, lotefa Teiti ${ }^{1,2}$, Bernard Mariamé ${ }^{2,3}$, Philippe Rochaix ${ }^{4}$, \\ Laurence Lamant ${ }^{1,4}$, Gilles Favre ${ }^{1,2,4}$, Véronique Maisongrosse ${ }^{4}$ and Anne-Françoise Tilkin-Mariamé ${ }^{\star, 1,2}$ \\ ${ }^{1}$ Unité INSERM UMR 1037, CRCT, F-31037 Toulouse, France; ${ }^{2}$ Université Paul Sabatier, F-31062 Toulouse, France; ${ }^{3}$ ITAV, USR 3505, \\ 1 Place Potier, F-31106 Toulouse, France and ${ }^{4}$ Service d'anatomo-Pathologie, Institut Universitaire du Cancer - IUCT, F-31059 \\ Toulouse, France
}

Background: CD70 is a costimulatory molecule of the tumour necrosis factor family expressed in activated immune cells and some solid tumours. In lymphocytes CD70 triggers T cell-mediated cytotoxicity and mitogen-activated protein kinase phosphorylation.

Methods: We evaluated the expression of CD70 in biopsies and melanoma cell lines. Using melanoma cell lines positive or not for CD70, we analysed CD70 function on melanoma progression.

Results: We report CD70 expression in human melanoma cell lines and tumour cells from melanoma biopsies. This expression was observed in $95 \%$ of primary melanomas but only $37 \%$ of metastases. Both monomeric and trimeric forms of CD70 were detected in tumour cell membrane fractions, whereas cytoplasmic fractions contained almost exclusively monomeric CD70. In vitro and in vivo experiments demonstrated that CD70 expression inhibited melanoma cell migration, invasion and pulmonary metastasis implantation independently of the tumour immune microenvironment. Increasing the levels of the trimeric form of CD70 through monoclonal antibody binding led to an increase in CD70 + melanoma cell invasiveness through MAPK pathway activation, RhoE overexpression, ROCK1 and MYPT1 phosphorylation decrease, and stress fibres and focal adhesions disappearance.

Conclusions: Our results describe a new non-immunological function of melanoma-expressed CD70, which involves melanoma invasiveness through MAPK pathway, RhoE and cytoskeletal modulation.

Melanomas arise from melanocytes and are the most aggressive and metastatic form of skin cancer. Melanoma progression results from the acquisition of molecular alterations that generate increasingly malignant phenotypes. Around 50\% of melanomas contain mutations in the serine/threonine kinase BRAF, with the BRAFV600E mutation being particularly common. Mutated BRAF induces constitutive activation of MAPK pathway, which is involved in crucial cellular processes such as proliferation and differentiation, and which favours tumour invasiveness (Liang et al, 2007; Ades and Metzger-Filho, 2012). Mutant BRAF also regulates RhoE (Rnd3) expression, which promotes cancer invasion through its action on actin cytoskeleton and focal adhesions (Klein and Aplin, 2009; Riou et al, 2010; Klein and
Higgins, 2011). The use of pharmacological inhibitors of MAPK pathway has shown promise for melanoma patients with mutated BRAF, however once resistance to these inhibitors occurs treatment options remain very limited (Ades and Metzger-Filho, 2012; Chopra and Nathan, 2015).

CD70 is a type II transmembrane costimulatory molecule, member of the TNF superfamily and expressed in activated NK, T and $\mathrm{B}$ lymphocytes in which it has important immunological roles through binding to its receptor, CD27 (Denoeud and Moser, 2011). CD70 is functional in its membranous homotrimeric form that, upon engagement, induces the trimerisation of CD27 receptor and the initiation of intracellular signalling. By itself CD70 also has an active role in $\mathrm{T}$ lymphocyte as $\mathrm{CD} 70$ crosslinking induced

*Correspondence: Dr A-F Tilkin-Mariamé; E-mail: anne-francoise.tilkin@inserm.fr

${ }^{5}$ Co-first authors.

Revised 20 August 2015; accepted 23 October 2015; published online 15 December 2015

(c) 2016 Cancer Research UK. All rights reserved 0007-0920/16 
by the binding of CD70-specific mAb QA32 has been shown to trigger $\mathrm{T}$ cell-mediated cytotoxicity and MAPK phosphorylation (Garcia et al, 2004). Some tumours express CD70, including lymphomas (Grewal, 2008), renal cell carcinomas and glioblastomas (Wischhusen et al, 2002).

In the present study, we reveal that CD70 is ectopically expressed in most primary human melanomas and that its expression then decreases and is often lost in the subsequent metastases. Interestingly, we found that CD70 expression markedly reduces pulmonary metastatic implantation, which was linked to the decreased migration and invasive capacities of CD70-positive melanoma cells and was independent of the tumour immune microenvironment. A CD70-specific mAb (QA32) is able to bind to CD70-positive melanoma cells, inducing a CD70 trimerisation enhancement and restoring tumour mobility. This was mediated through overactivation of MAPK pathway and overexpression of RhoE, leading to the disappearance of stress fibres and focal adhesions.

\section{MATERIALS AND METHODS}

Tumour cell lines and mice. Human melanoma cell lines LB1319-MEL (wild-type NRAS and BRAF) and LB39-MEL (wild-type NRAS and mutated BRAFV600E), and three melanoma cell lines derived from the same patient LB33-MEL (all wild-type NRAS and BRAF) - LB33-MEL.A (cutaneous metastasis developed in 1988), LB33-MEL.B (lymph node metastasis developed in 1993) and LB33-MEL.D (intestinal metastasis developed in 1999) - were provided by Prof. Boon (LICR, Brussels). B16F10-CD70 and its control cell line B16F10-wt were produced in our laboratory (Cormary et al, 2005) by genetic modification of B16F10 murine melanoma cells purchased from the ATCC. All tumour cells were maintained in RPMI 1640/10\% FCS.

Six- to eight-week-old female C57BL/6 and NMRI nu/nu mice (Elevages Janvier) and C57BL/6 IFN- $\gamma$ KO mice produced in our animal facility (agreement no.B.31.555.26) were used for in vivo experiments. All experiments involving mice were done using appropriate conditions, supervised by Regional Ethic Committee of Midi-Pyrénées (agreement ICR-2009-0011 and ICR-2009-0020).

Antibodies. For immunohistochemistry (IHC), two CD70-specific $\mathrm{mAbs}$ were used. The anti-CD70 QA32 hybridoma supernatant (Garcia et al, 2004) and the anti-CD70 mAb clone Ki-24 from BD Biosciences (San Jose, CA, USA). MelanA and KBA.62 were also detected with specific mAbs ready to use from Dako (Glostrup, Denmark). For flow cytometry and cell sorting, PE-conjugated anti-CD70 $\mathrm{mAb}$ and control isotype (BD Biosciences) were used. Western blot analyses used primary Abs against CD70, MEK, P-MEK, ERK, P-ERK, RhoE, ROCK1, ROCK2, phospho-MYPT1 and total MYPT1 (Tebu-Bio, Le Perray En Yvelines, France; Santa Cruz Biotechnology, Dallas, TX, USA; Ozyme, Montigny-leBretonneux, France and Cell Signaling, Danvers, MA, USA) and HRP-labelled secondary Ab (R\&D Systems, Minneapolis, MN, USA). For all different invasion and migration assays and immunofluorescence staining, Ctrl Ig was Y3 hybridoma supernatant diluted to half and the anti-CD70 mAb was QA32 hybridoma supernatant diluted to half. The anti-vinculin, the anti-GAM-AlexaFluor488 $\mathrm{Ab}$ and the phalloidin-AlexaFluor594 were from Sigma (St Louis, MO, USA).

Immunohistochemical detection in melanoma tumour specimens. Two methods were used to detect CD70 in melanoma tumour samples:

- IHC was performed on 4 - $\mu$ m-thick routinely processed formalin-fixed paraffin-embedded sections of tumour specimens with neighbouring normal tissue. A heat-induced epitope retrieval technique in a citrate buffer ( $\mathrm{pH}$ 6) was first performed. The CD70-specific mAb (QA32) was incubated for $30 \mathrm{~min}$ and revealed using EnVision G|2 System/AP (Dako) enzymeconjugated polymer backbone according to the manufacturer's protocol and visualised by Permanent Red Chromogen (Fas Red) included in the kit. MelanA and KBA.62 were revealed by FLEX/HRP (20-min incubation), FLEX DAB + Sub-Chromo (10-min incubation) and FLEX Hematoxylin (5-min incubation). The IHC was processed using an Autostainer plus (Dako) slide processor.

- Stainings were also performed on cryostat sections, fixed and dried before incubation with anti-CD70 mAb (BD Biosciences; 1:50 TBS plus 5\% BSA) overnight at $4{ }^{\circ} \mathrm{C}$ and then with secondary biotinylated goat anti-mouse Ab (KIT Dako; $1: 200$ ) for $30 \mathrm{~min}$. Bound $\mathrm{Ab}$ was detected with streptavidin-alkaline phosphatase kit (Vector, Burlingame, CA, USA) at room temperature in humidified chamber. Photos were taken with a DM 3000 microscope (Leica Microsystems, Wetzlar, Germany) and a CMS GmbH DFC 295 camera (Leica Microsystems). The slides were subsequently counterstained with hemalun.

In melanoma specimens, $2 \%$ of CD70 + tumour cells were used as threshold value, because detection of rare and isolated CD70 + tumour cells was often observed in $<2 \%$ of melanoma cells that we considered as not significant. For each tumour specimen, a slide was totally analysed by a pathologist and a biologist, independently and blinded. Loco-regional differences between epidermal and dermal CD70 expression were observed in most specimens and were considered.

Local ethic committee approved the analysis of human melanomas (agreement ICRCRB DC-2011-1364).

Flow cytometric analyses. Membrane CD70 expression was analysed on a BD FACSCalibur (San Jose, CA, USA). To compare several analyses, index of specific fluorescence was calculated: (median fluorescence intensity (MFI) with the specific antibody MFI with the isotype control)/MFI with the isotype control $\times 100$.

Selection of LB39-MEL CD70-positive and CD70-negative clones. LB39-MEL cells were stained with anti-CD70 mAb. $\mathrm{CD} 70+$ and $\mathrm{CD} 70-$ subpopulations were first sorted with Becton Dickinson FACS Aria II (San Jose, CA, USA). Then a cellular cloning allowed the isolation of LB39-MEL CD70 + and CD70 - clones.

Western blot analyses. Total protein extracts were prepared by the standard procedure. Proteins were then separated on SDSPAGE gel electrophoresis and blotted onto PVDF membranes (GE Healthcare, Velizy-Villacoublay, France or Pittsburgh, PA, USA). The filters were incubated at $4{ }^{\circ} \mathrm{C}$ overnight with primary $\mathrm{Abs}$, then with secondary $\mathrm{Ab}$ for $1 \mathrm{~h}$ at room temperature. A chemiluminescence detection ECL kit (Thermo Scientific Pierce, Waltham, MA, USA) was used, and band intensities were quantified using ImageJ software (National Institutes of Health, Bethesda, MD, USA). Actin was used as a loading control (Chemicon, Rolling Meadows, IL, USA).

Cytosolic and membrane fractions were prepared using the plasma membrane protein extraction kit (Biovision Incorporated, Milpitas, CA, USA) according to the manufacturer's protocol before western blot analysis.

Pulmonary metastases implantation. All mice strains were injected i.v. with B16F10-wt or B16F10-CD70 cells $\left(1 \times 10^{5}\right)$. Mice were killed 10 days later. Macroscopic metastases were detected visually and quantified. The NMRI nu/nu mice were also injected i.v. with LB1319-MEL cells transfected with siCtrl or siCD70 $\left(1 \times 10^{6}\right)$. Mice were killed 30 days later. The lungs were fixed in formalin and paraffin-embedded to visualise microscopic metastases. 
Photos were taken with a DMR microscope (Leica Microsystems) and a DS-Fil camera (Nikon Instruments, Amsterdam, Netherlands). The experiments included 3-5 mice per group and were repeated twice.

Transfection of siRNAs. LB1319-MEL melanoma cells were transiently transfected with siRNAs, as previously described (Tilkin-Mariame et al, 2005). Briefly, $5 \times 10^{5}$ cells were transfected with $2.5 \mathrm{~nm}$ siRNA using JetPrime (Polyplus Transfection, Illkirch, France). The following siRNA duplexes, purchased from Thermo Fisher (Waltham, MA, USA), were used: siCD70 pool (CACCAAG GUUGUACCAUUG, GCAUCUACAUGGUACACAU, GCAGCU GAAUCACACAGGA and UGACCACUGCUGCUGAUUA); and a non-targeting siNegative pool (siNeg) (UGGUUUACAUGUC GACUAA, UGGUUUACAUGUUGUGUGA, UGGUUUACAU GUUUUCUGA and UGGUUUACAUGUUUUCCUA).

\section{Cell migration and invasion in vitro assays}

- Migration and invasion assays were performed with Ctrl Ig or anti-CD70 mAb, using triplicate or quadruplicate wells. For migration assays $2.5 \times 10^{4}$ cells were added in RPMI 1640/2\% FCS plus Abs in the upper compartment of a $8-\mu \mathrm{m}$ pore size transwell system (BD Biosciences). The bottom chamber was filled with RPMI 1640/10\% FCS. After $24 \mathrm{~h}$, cells on the bottom surface of the filter were stained and counted. The number of control cells in the lower chamber in presence of Ctrl Ig was considered as $100 \%$. The protocol is similar for invasion assays, but invasion chambers (BD Biosciences) were used and the cells were counted $48 \mathrm{~h}$ later.

- Wound-healing assays: $2.5 \times 10^{4}$ cells were plated on collagen type I (5 mg cm ${ }^{-2}, \mathrm{BD}$ Biosciences) and treated with the Abs. Two days later, a cleared section of a confluent cell monolayer was produced by scratching. Photos were taken with an Eclipse Ti microscope (Nikon Instruments) and a CoolSNAP HQ2 camera (Photometrics, Tucson, AZ, USA) in three randomised fields.

Three-dimensional spheroid invasion. Formation of 3D spheroids occurred after $72 \mathrm{~h}$ at $37^{\circ} \mathrm{C}$ using $1 \times 10^{3}$ LB1319-MEL cells on 96-well plate coated with $1 \%$ agarose (Interchim, Montluçon, France). Spheroids were then embedded into a bovine collagen I gel containing EMEM, L-glutamine and 2\% FBS with the Abs for $24 \mathrm{~h}$. Then MEK inhibitor U0126 (Calbiochem) was added or not at $5 \mu \mathrm{M}$. Spheroids sizes were measured and photos were taken after $72 \mathrm{~h}$ using an Eclipse Ti microscope (Nikon Instruments) and a CoolSNAP HQ2 camera (Photometrics).

Immunofluorescence staining of stress fibres and focal adhesions. Melanoma cells $\left(2.5 \times 10^{4}\right)$ were plated on poly-lysin $(0.001 \%$, Sigma)-coated coverslips in six-well plates and treated with the Abs. After 24 or 48 h, cells were fixed with cold Cristal, vinculin and actin were labelled. Coverslips were mounted on slides using ProLong/DAPI (Invitrogen, Carlsbad, CA, USA). After an overnight incubation at $4{ }^{\circ} \mathrm{C}$, photos were taken using an Eclipse $\mathrm{Ti}$ microscope and a CoolSNAP HQ2 camera in three randomised fields.

Statistical analysis. Statistical analyses were performed using GraphPad Prism software (San Diego, CA, USA). Significance of analyses was assessed by $t$-test, Mann-Whitney test, $\chi^{2}$-test or Tukey one-way ANOVA test. All statistical tests were two sided. The values are expressed as means \pm s.d. in the figures. $P$-values under 0.05 were considered statistically significant.
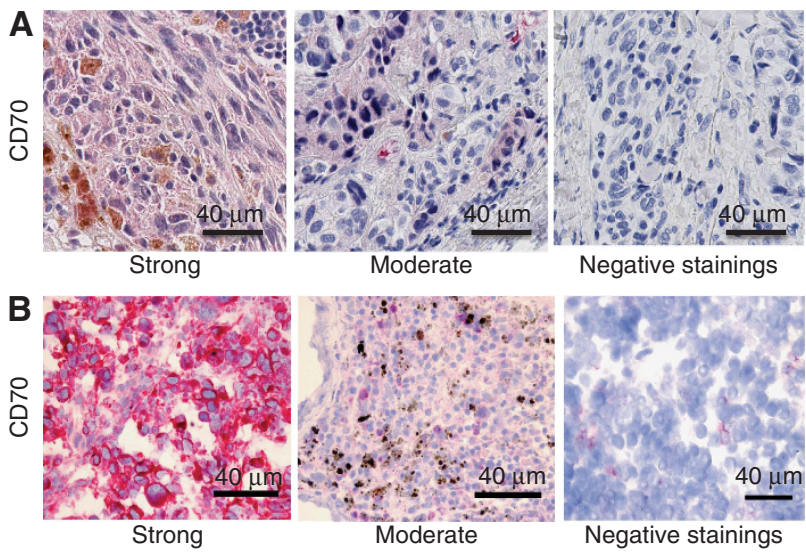

Negative stainings

C
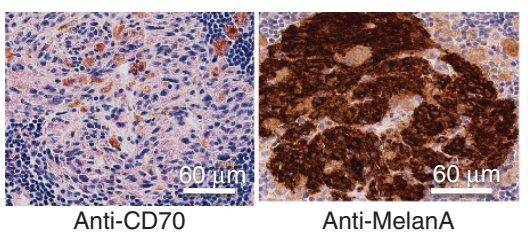

D
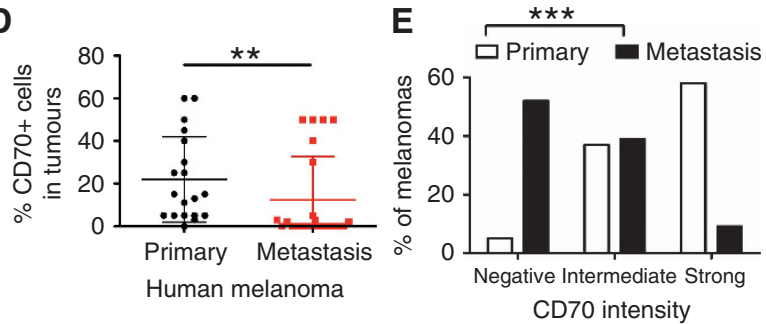

Figure 1. CD70 ectopic expression in human melanomas.

Representative images of CD70 expression after staining by IHC paraffin-embedded melanoma human biopsies (A) or staining of cryostat sections of frozen melanoma tumour specimens (B). Negative staining corresponded to CD70 expression in $<2 \%$ of melanoma cells, moderate staining in $2-20 \%$ and strong staining in more than $20 \%$. (C) Three slides of the same melanoma fragment showing that CD70 expression is in the tumour cells. The slides were labelled with antiCD70 mAb, anti-MelanA or anti-KBA.62 antitumour antibodies.

(D) Primary human melanomas contain more CD70 + tumour cells than metastases as quantified in paraffin-embedded melanoma biopsies. (E) The intensity of CD70 expression is also stronger in primary melanomas than in metastases. $P$-values were evaluated using the Mann-Whitney test (D) or the $\chi^{2}$-test $(\mathbf{E}) .{ }^{\star \star} P<0.005 ;{ }^{\star \star \star} P<0.001$.

\section{RESULTS}

CD70 ectopic expression in melanomas. To assess CD70 expression in human melanoma cells, IHC was used to screen 19 primary and 24 metastatic melanoma specimens. CD70 expression was detected with two CD70-specific mAbs (Figure 1A-C). Specimens of healthy skin were also stained as negative controls (Supplementary Figure 1). CD70 was mainly detected in the cytosol of the tumour cells. Further analysis of CD70 expression showed that $95 \%$ (18 out of 19) of primary tumours $v s 37 \%$ (9 out of 24) of the skin and lymph node metastases contained a significant $(>2 \%)$ fraction of $\mathrm{CD} 70+$ tumour cells. Primary melanomas also showed a significantly higher percentage of $\mathrm{CD} 70+$ tumour cells (Figure 1D) and a significant increase in the expression levels of CD70, although CD70 expression was variable among the different specimens (Figure 1E).

This CD70 ectopic expression was also observed in four human melanoma cell lines using flow cytometry. The LB1319-MEL line was strongly and homogenously $\mathrm{CD} 70+$, whilst another cell line 
LB39-MEL had a subpopulation of $20 \%$ of cells expressing CD70 (Figure 2A). CD70 + and CD70 - clones were isolated from the LB39-MEL cell line (Figure 2A) and their CD70 expression remained stable for more than 10 passages. To monitor CD70 expression over the course of disease progression, we analysed three melanoma cell lines that had been derived from three relapses of the same patient, at different times and from different metastatic localisations: LB33-MEL.A a cutaneous metastasis; LB33-MEL.B a lymph node metastasis that appeared 5 years later; and LB33-MEL.D an intestinal metastasis that appeared a further 6 years later (Chiari et al, 2000). The proportion of $\mathrm{CD} 70+$ cells in these cells is, respectively, $50 \%, 5 \%$ and $0 \%$,
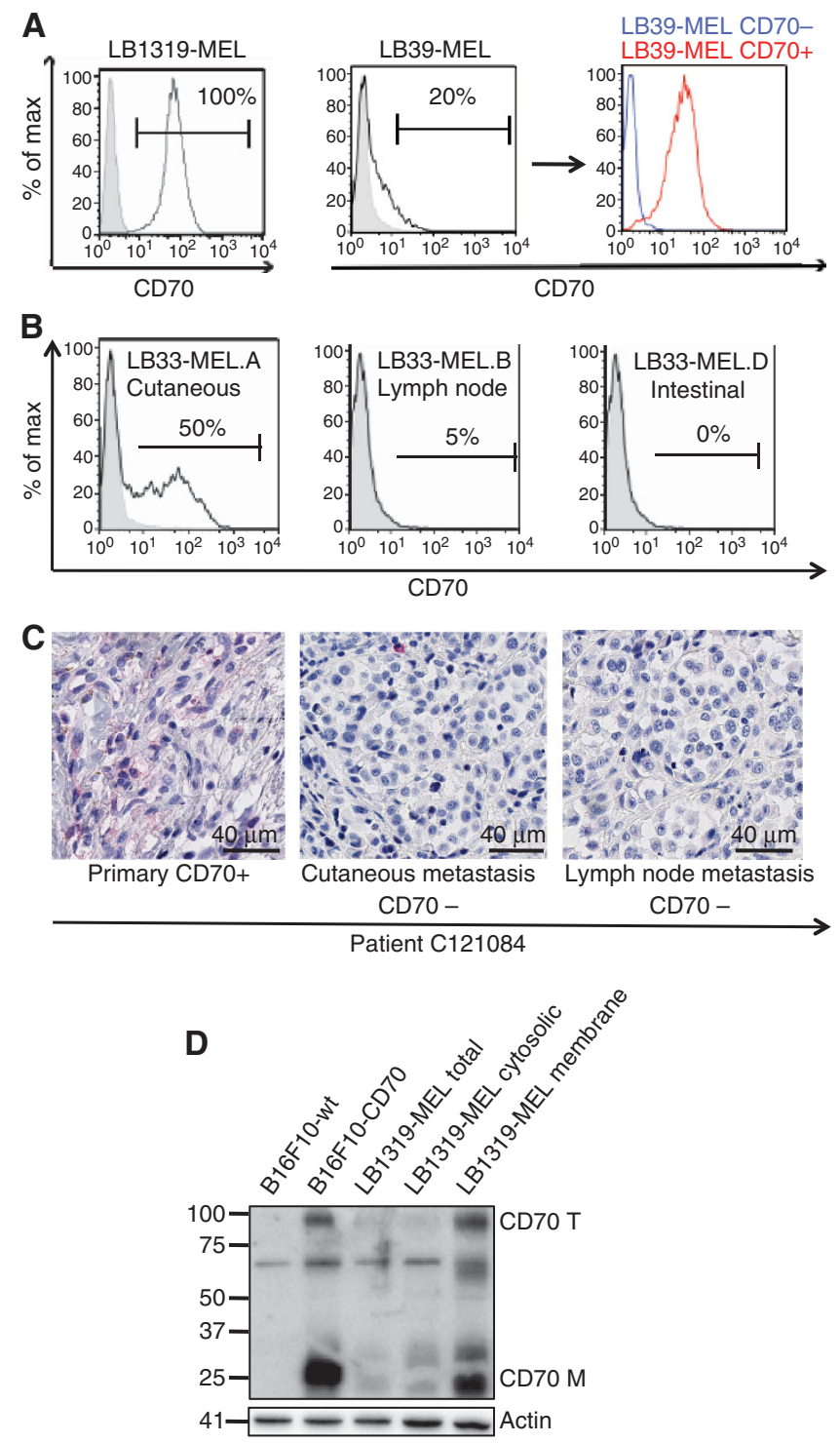

Figure 2. CD70 expression decreases with time and disease progression in human melanomas. (A) CD70 membrane expression measured by flow cytometry in three representative melanoma cell lines: LB1319-MEL; LB39-MEL; and LB39-MEL clones CD70 + vs CD70 - . (B) Decrease of membrane CD70 expression in three melanoma cell lines derived from the same patient: LB33-MEL.A (skin, 1988); LB33-MEL.B (lymph node, 1993); and LB33-MEL.D (intestine, 1999). (C) Decrease with disease progression of melanoma-expressed CD70: primary tumours, skin and lymph node metastases from the same patient C121084. (D) Monomeric (CD70 M) and trimeric (CD70 T) forms of CD70 detected by western blot in LB1319-MEL (total, cytosolic and membrane fractions), B16F10-wt and B16F10-CD70 cells. showing that CD70 expression decreased and even disappeared over time as the disease progressed (Figure $2 \mathrm{~B}$ ). This result was also observed using IHC analysis of two other patients (Figure 2C and data not shown).

The sub-cellular localisation of CD70 in melanoma cells was analysed by western blotting of the sub-cellular fractions of LB1319-MEL cells. Both the monomeric and trimeric forms of CD70 were detected in the global fraction, indicating that these CD70 molecules were functional. Both forms were also detected in the membranous fraction, whereas the cytosolic fraction contained almost exclusively the monomeric form (Figure 2D). Moreover, the monomeric and trimeric forms were also expressed in murine cell line developed by Cormary et al (Figure 2D).

Altogether, these data showed that most human primary melanomas express CD70 and that its levels tend to decrease in metastasis.

CD70 expression reduces the metastatic capacity of melanoma cells. The genetically modified murine melanoma cell lines B16F10-wt and B16F10-CD70 (Cormary et al, 2005) were subcutaneously injected into three murine models: C57BL/6 immunocompetent mice; C57BL/6-IFN- $\gamma \mathrm{KO}$; and NMRI nu/nu immunocompromised mice. In all three models, CD70 + and CD70 - tumours grew locally at similar rates (Supplementary Figure 2A). On the contrary, when these cells were intravenously injected, all three models showed significantly less metastases in the lungs of mice injected with CD70 + melanoma cells (Figure 3A
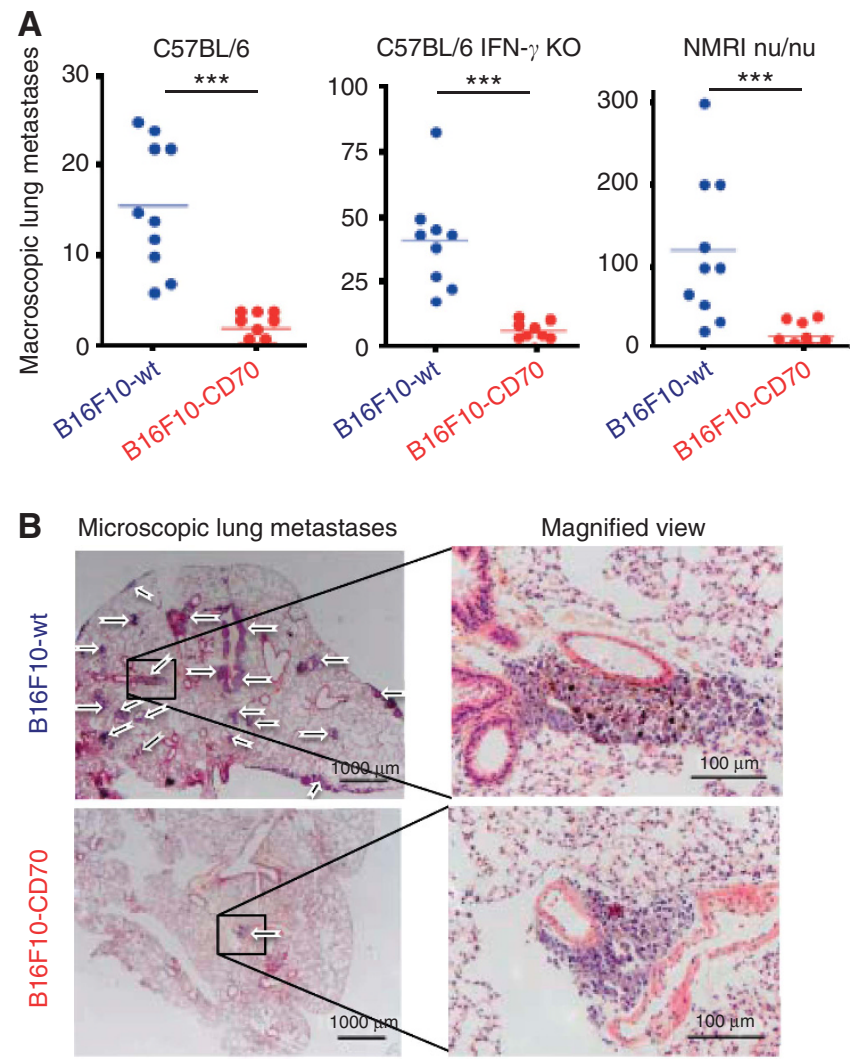

Figure 3. $\mathrm{CD} 70$ expression is associated with decreased in vivo metastatic capacity. (A) C57BL/6, C57BL/6 IFN- $\gamma$ KO and NMRI nu/nu mice were injected i.v. with B16F10-wt (blue) or B16F10-CD70 (red) cells and pulmonary metastases were quantified, showing that B16F10CD70 cells induced less metastases implantations. (B) Representative lung photomicrographs (left) and enlargement of metastatic areas (right) of C57BL/6 mice injected i.v. with B16F10-wt (top) or B16F10CD70 (bottom) cells. Arrows indicate metastases. $P$-values were evaluated using the t-test. ${ }^{\star \star \star} P<0.001$. 
and B), showing that CD70 expression decreased the metastatic implantation capacity of the melanoma cells.

This decrease in the metastatic implantation potential of CD70expressing melanoma cells was confirmed using human CD70 + melanoma cells. First we controlled that CD70 expression or CD70 silencing did not inhibit tumour cell proliferation in vitro (Supplementary Figure 2B). Then lung metastasis implantation was tested with transfected LB1319-MEL cells. No lung metastases appeared after i.v. injection of CD70 + LB1319-MEL cells (wt and siCtrl) into NMRI nu/nu mice (Supplementary Figure 3A and B), whereas siRNA-mediated CD70 silencing (siCD70; Supplementary Figure $3 \mathrm{~A}$ ) in these melanoma cells before i.v. injection induced lung metastasis development as shown in Supplementary Figure 3B and illustrated in Supplementary Figure 3C.

If we compare the number of metastases induced by the B16F10-wt vs the B16F10-CD70 cells in the three murine models (Figure 3A), it is obvious that the immunocompromised mice developed more metastases than the immunocompetent mice, suggesting that immune effector cells of the tumour microenvironment, such as T lymphocytes and IFN $\gamma$-secreting cells, partially controlled metastatic lung colonisation. However, the seven-fold decrease in the number of lung metastases in mice intravenously injected with $\mathrm{CD} 70+$ cells compared with $\mathrm{CD} 70$ - cells was similar for all three mouse models, confirming that CD70 reduces metastatic implantation efficacy by a mechanism that is independent of the tumour immune microenvironment. Therefore, we hypothesised that this decrease in metastatic potential was due to an intrinsic property of CD70 within melanoma cells.

CD70 reduces melanoma cell migration and invasion capacities in vitro. To elucidate the mechanism involved in the CD70mediated metastatic reduction, $\mathrm{CD} 70+$ and CD70 - murine and human melanoma cells were used to study in vitro migration and invasion. Wound-healing, transwell and Boyden chamber assays showed that CD70 expression inhibited B16F10 melanoma migration (Figure 4A and C) and invasion (Figure 4B). Similar experiments with human melanoma cells also confirmed that CD70 expression significantly inhibited migration (Figure 4D) and invasion (Figure 4E). Interestingly, addition of the CD70-specific mAb QA32 to the culture medium reversed the inhibition of
A

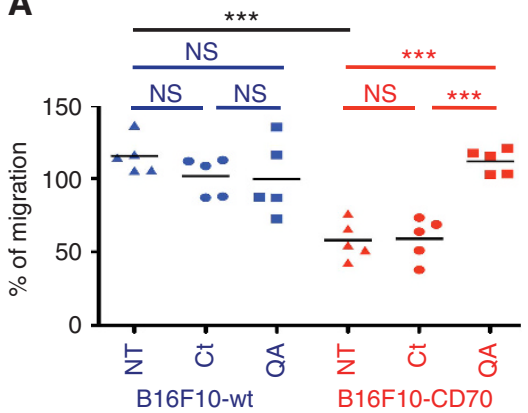

C
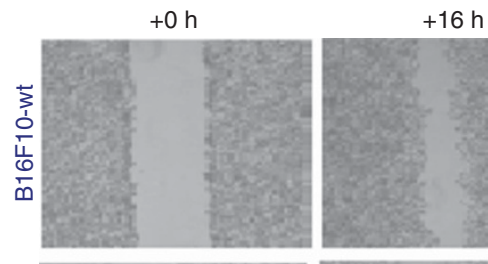

B

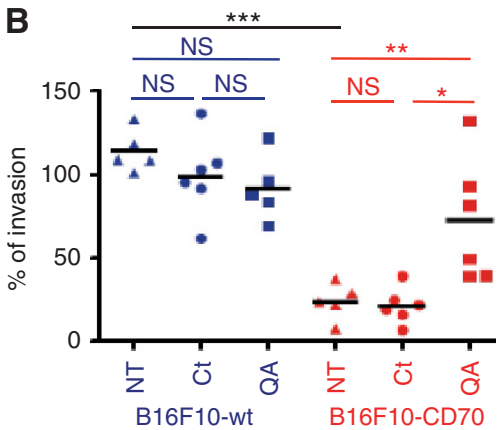

$+24 \mathrm{~h}$

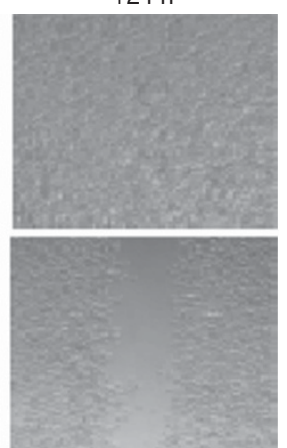

D

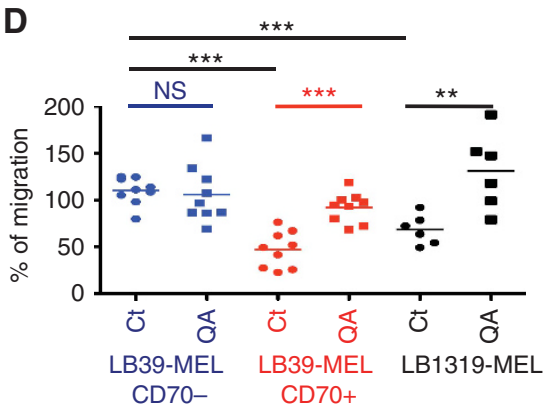

E

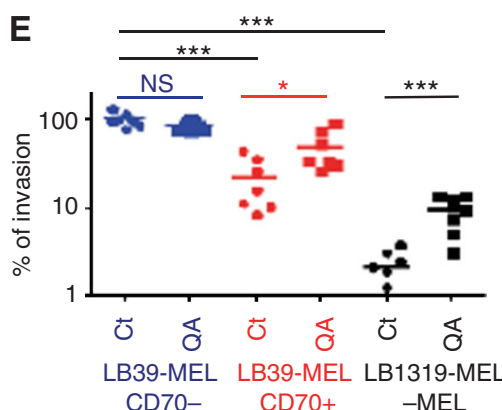

Figure 4. In melanoma cells CD70 expression is associated with decreased in vitro migration and invasion capacities. Decreased in vitro migration (A) and invasion (B) capacities of B16F10-CD70 + cells analysed using, respectively, transwells and invasion chambers with B16F10-wt vs B16F10-CD70 cells. In the upper chamber the medium was or not (NT) supplemented with Ctrl Ig (Ct) or anti-CD70 mAb (QA). Decreased in vitro migration capacity of B16F10-CD70 + cells was analysed by wound-healing experiments done with B16F10-wt and B16F10-CD70 cells (C).

Decreased in vitro migration (D) and invasion (E) capacities of CD70 + human cells: LB39-MEL CD70 - clone (blue), LB39-MEL CD70 + clone (red) and LB1319-MEL cells (black). Results (A, B, D and E) are expressed as mean values $(n=7)$. P-values were evaluated using the Tukey ANOVA test. ${ }^{\star} P<0.05 ;{ }^{* \star} P<0.005 ;{ }^{* \star \star} P<0.001$. 
migration and invasion (Figure 4A, B, D and E). This QA32 mAb is known to crosslink CD70 molecules and activates a signalling pathway in T lymphocytes (Garcia et al, 2004).

As these experiments were done in vitro with melanoma cells alone, they show that the role of CD70 in migration and invasion is intrinsic, and can be modulated by inducing CD70 signalling via the anti-CD70 mAb QA32.

Anti-CD70 mAb binding favours the trimeric CD70 form and induces melanoma cell invasion through MAPK pathway activation, RhoE overexpression, ROCK1 inhibition and cytoskeleton modifications in vitro. As CD70 expression correlates with poor migration and invasive capacities, which were restored with anti-CD70 (QA32) binding, we hypothesised that QA32 binding was able to modify the melanoma-expressed CD70 to induce a signalling pathway involved in melanoma invasion and metastasis. Western blot analyses showed that the addition of
QA32 to the culture medium significantly increased the ratio between the trimeric and monomeric forms of CD70 (Figure 5A and B). Therefore, we hypothesised that the trimeric form of CD70 positively regulated a signalling pathway involved in melanoma migration and invasion. Previous publications have suggested that this signalling pathway could include the MAPK pathway as antiCD70 mAb binding was shown to induce the activation of the MAPK pathway in EBV-transformed B cells and in CD70 + T lymphocytes (Garcia et al, 2004; Park et al, 2010). In addition, the MAPK pathway is known to regulate migration, invasion and metastasis in several tumour models, including melanomas (Liang et al, 2007; Dua and Gude, 2008). Western blot analyses of CD70 + melanoma cells cultured in the presence of QA32 confirmed that addition of this $\mathrm{mAb}$ activated the MAPK pathway, as an eight-fold increase in ERK1 and ERK2 phosphorylation was observed in LB1319-MEL cells (Figure 5C) and a three-fold increase in MEK1 phosphorylation was observed in LB39-MEL
A

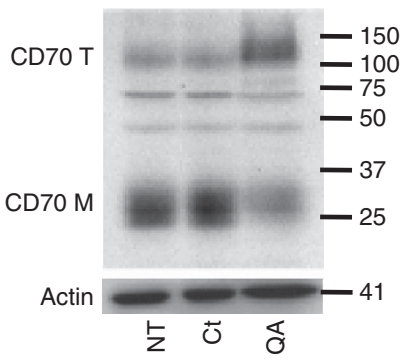

D
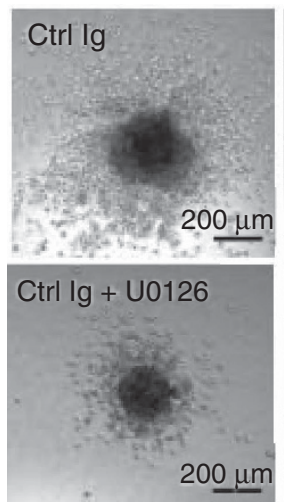

$200 \mu \mathrm{m}$

$\mathbf{F}$
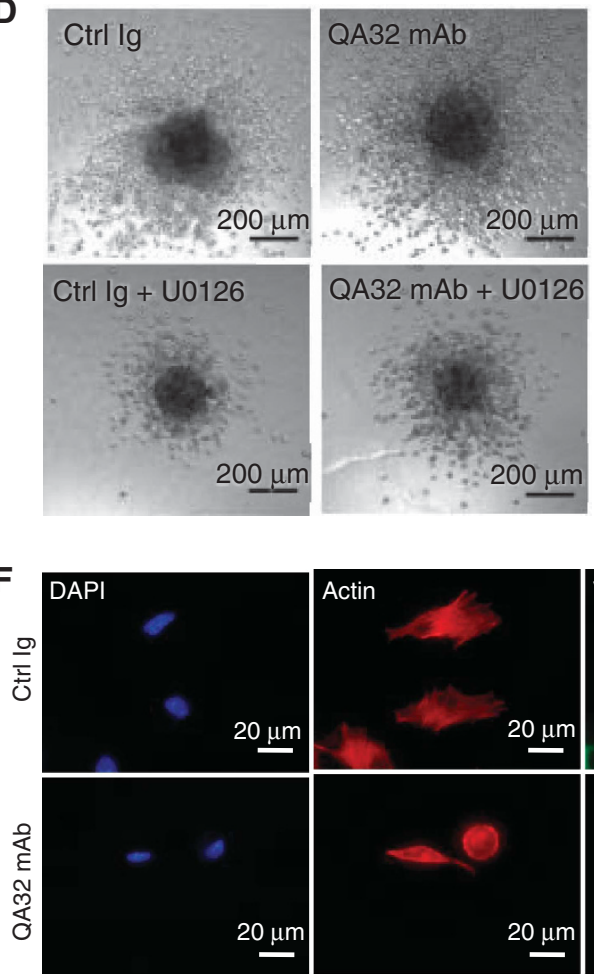

B

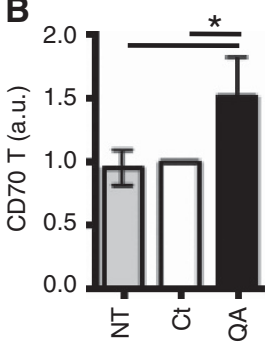

C

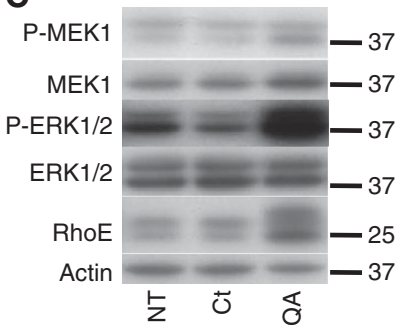

$\mathbf{E}$
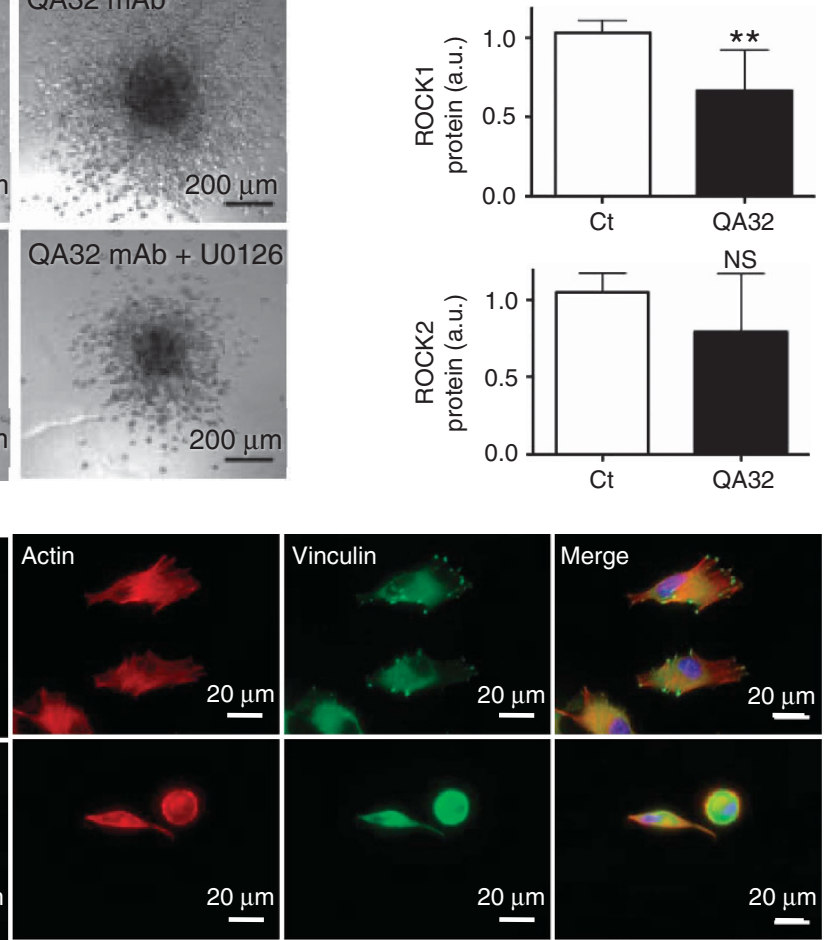

Figure 5. CD70 signalling modulates MAPK activation, RhoE and ROCK1 expression, and cytoskeleton formation. Western blot analysis (A) and quantification (B) in arbitrary units (a.u.) of CD70 M decrease and CD70 T increase in LB1319-MEL cells incubated with anti-CD70 mAb (QA). Controls are untreated (NT) and Ctrl Ig (Ct)-incubated LB1319-MEL cells. (C) Western blots analyses of the MEK/ERK pathway activation and RhoE overexpression in these LB1319-MEL cells. (D) The 3D invasion using LB1319-MEL spheroids treated with the Abs plus MEK inhibitor U0126, showing anti-CD70-induced invasion enhancement and U0126-induced invasion inhibition. (E) Western blot quantification of total ROCK1 and ROCK2 in LB1319-MEL cells incubated with Ctrl or anti-CD70 mAb, showing anti-CD70-induced ROCK1 inhibition. (F) Disappearance of stress fibres (actin) and focal adhesions (vinculin) in LB1319-MEL cells treated with anti-CD70 mAb for $48 \mathrm{~h}$. Results in $\mathbf{B}$ and $\mathbf{E}$ are expressed as mean values $(n=3)$. $P$-values were evaluated using the Tukey ANOVA test $(\mathbf{B})$ or $t$-test $(\mathbf{E}) .{ }^{* \star} P<0.005$. 
cells (Supplementary Figure 4A). Furthermore, in a 3D spheroid model, the invasive capacity of $\mathrm{CD} 70+$ melanoma cells was enhanced by QA32 and was reduced in the presence of the MEK inhibitor U0126 (Figure 5D and Supplementary Figure 4B). These results show that in melanoma cells the trimeric form of CD70, unlike the monomeric form, positively regulates the pro-invasive and pro-metastatic MAPK pathway, and that it exerts its action upstream of MEK as melanoma cell invasion was reduced in the presence of both QA32 and U0126 inhibitor. In addition, this trimeric CD70-induced MAPK pathway upregulation is independent of the presence of a mutation in BRAF gene at V600 locus, as it was observed in LB39-MEL cells, which possess BRAFV600E mutated proteins (Supplementary Figure 4A), and in LB1319-MEL cells, which are not mutated at this locus (Figure 5A and B).

Cell invasion is also influenced by the actin cytoskeleton and by focal adhesions, both of which are essential for cell movement and are regulated by several Rho GTPases. RhoA, B and C positively regulate the cytoskeleton via multiple effectors, including the Rho kinases ROCK1/2 (Vega and Ridley, 2008) through MYPT1 phosphorylation (Amano et al, 1996), whereas RhoE antagonises Rho/ROCK signalling and disrupts stress fibres and focal adhesions (Chardin, 2006). Consequently, RhoE can promote tumour invasion through its effect on the actin cytoskeleton. In human melanoma cells, it has been shown that BRAF controls RhoE activity and that, when BRAF is inhibited, RhoE activity is also decreased, leading to an enhanced number of stress fibres and focal adhesions, and therefore decreased cell invasion (Klein et al, 2008). We evaluated the effect of QA32 binding to CD70 + melanoma cells on RhoE, ROCK1/2, MYPT1, stress fibres and focal adhesions. Results show a two-fold upregulation in RhoE overall levels (Figure 5C and Supplementary Figure 4A). As expected, a decrease in ROCK1 and MYPT1 phosphorylation was detected (Figure 5E and Supplementary Figure 4C and D) and a marked decrease in stress fibres and focal adhesions was also detected in LB1319-MEL cells after only $24 \mathrm{~h}$ (Supplementary Figure 4E) and maintained after $48 \mathrm{~h}$ (Figure 5F). Similar results were obtained with LB39MEL CD70 + cells (data not shown).

These findings reveal that in melanoma cells, the presence of cytosolic monomeric CD70 correlates with poor invasion and metastatic capacities, whereas the binding of the anti-CD70 $\mathrm{mAb}$ QA32 enhances the level of trimeric CD70, which activates the MAPK/RhoE signalling pathway leading to a reduction in ROCK1, MYPT1 phosphorylation, stress fibres and focal adhesions, in turn enhancing melanoma invasiveness.

\section{DISCUSSION}

This work has described that CD70 is expressed in human melanoma cells, that its expression is reduced in metastases and that its role in regulating melanoma invasion and metastasis is independent of the tumour immune microenvironment. As an increased metastatic potential is associated with both the decreased levels of monomeric CD70 and CD70 silencing, melanomaexpressed CD70 could become a useful prognostic marker.

CD70 expression has already been reported in renal cell carcinomas, glioblastomas, astrocytomas and ovarian cancers. In addition, Ryan et al (2010) previously reported that $16 \%$ of melanoma samples expressed CD70, although they did not describe the metastatic status of their sample. Here we describe CD70 expression both in human melanoma cell lines and in tumour cells from melanoma biopsies. Furthermore, we analysed primary and metastatic tumour samples separately: the number of CD70 + cells and the level of CD70 expression are significantly decreased in metastases. This loss of CD70 expression during disease progression was explained by our in vitro experiments, which showed that $\mathrm{CD} 70+$ melanoma cells have a reduced capacity to migrate and invade, meaning they presumably had a limited ability for implantation of lung metastases.

The observation that $\mathrm{CD} 70+$ melanoma cell invasion is reduced in vitro in the absence of a tumour immune microenvironment has proven that this effect is intrinsic within the CD70+ tumour cells. Besides this invasion, inhibition is not due to interactions between the CD70 ligand and its receptor CD27 on melanoma cells, as CD27 is neither expressed nor induced by antiCD70 mAb binding (Supplementary Figure 5A).

We found that CD70 is not only a ligand but also has its own signalling depending on its oligomerisation: monomeric CD70 limits melanoma cell migration and invasion but the trimeric form, induced by anti-CD70 $\mathrm{mAb}$ binding, restores these activities via MAPK activation, RhoE overexpression and inhibition of ROCK1, MYPT1 phosphorylation, stress fibres and focal adhesion. These differences could be the result of a different conformation of CD70 that could change the protein-protein interactions and/or its localisation on the membrane (i.e., lipidic rafts). These CD70 dramatic specificities need further studies to be fully understood. For now, we performed a real-time quantitative PCR array for genes involved in cell motility. Interestingly, this approach confirmed the QA32-induced RhoE overexpression and showed that three other genes (VEGF, VASP and PTPN1) were also significantly increased (Supplementary Figure $5 \mathrm{~B}$ and $\mathrm{C}$ ). These genes are already known for their involvement in tumour invasion: VEGF role is well characterised in angiogenesis and metastases (Ni et al, 2012); VASP has been shown to regulate stress fibres and migration (Chen et al, 2014); and PTPN1 promotes prostate cancer progression (Lessard et al, 2012).

Finally, several clinical trials are in progress to test the antitumor effects of anti-CD70 antibodies (Grewal, 2008). Our data strengthen the role of CD70 trimerisation in tumour invasion and suggest that anti-CD70 antibodies should be carefully selected to eliminate those that promote tumour invasion and metastasis, through CD70 trimerisation.

\section{ACKNOWLEDGEMENTS}

We thank Prof. Lopez-Botet for providing the anti-CD70 QA32 hybridoma and Prof. Boon for the human melanoma cell lines. This work was supported by INSERM and Institut Claudius Regaud and by grants from Ligue Contre le Cancer, Association pour la Recherche Contre le Cancer and Ministère de l'Enseignement Supérieur et de la Recherche.

\section{CONFLICT OF INTEREST}

The authors declare no conflict of interest.

\section{REFERENCES}

Ades F, Metzger-Filho O (2012) Targeting the cellular signaling: BRAF inhibition and beyond for the treatment of metastatic malignant melanoma. Dermatol Res Pract 2012: 259170.

Amano M, Ito M, Kimura K, Fukata Y, Chihara K, Nakano T, Matsuura Y, Kaibuchi K (1996) Phosphorylation and activation of myosin by Rho-associated kinase (Rho-kinase). J Biol Chem 271: 20246-20249.

Chardin P (2006) Function and regulation of Rnd proteins. Nat Rev Mol Cell Biol 7: 54-62.

Chen XJ, Squarr AJ, Stephan R, Chen B, Higgins TE, Barry DJ, Martin MC, Rosen MK, Bogdan S, Way M (2014) Ena/VASP proteins cooperate with the WAVE complex to regulate the actin cytoskeleton. Dev Cell 30: 569-584. Chiari R, Hames G, Stroobant V, Texier C, Maillere B, Boon T, Coulie PG (2000) Identification of a tumor-specific shared antigen derived from an 
Eph receptor and presented to CD4 T cells on HLA class II molecules. Cancer Res 60: 4855-4863.

Chopra N, Nathan PD (2015) Trametinib in metastatic melanoma. Expert Rev Anticancer Ther 15: 749-760.

Cormary C, Hiver E, Mariame B, Favre G, Tilkin-Mariame AF (2005) Coexpression of CD40L and CD70 by semiallogenic tumor cells induces anti-tumor immunity. Cancer Gene Ther 12: 963-972.

Denoeud J, Moser M (2011) Role of CD27/CD70 pathway of activation in immunity and tolerance. J Leukoc Biol 89: 195-203.

Dua P, Gude RP (2008) Pentoxifylline impedes migration in B16F10 melanoma by modulating Rho GTPase activity and actin organisation. Eur J Cancer 44: 1587-1595.

Garcia P, De Heredia AB, Bellon T, Carpio E, Llano M, Caparros E, Aparicio P, Lopez-Botet M (2004) Signalling via CD70, a member of the TNF family, regulates T cell functions. J Leukoc Biol 76: 263-270.

Grewal IS (2008) CD70 as a therapeutic target in human malignancies. Expert Opin Ther Targets 12: 341-351.

Klein RM, Aplin AE (2009) Rnd3 regulation of the actin cytoskeleton promotes melanoma migration and invasive outgrowth in three dimensions. Cancer Res 69: 2224-2233.

Klein RM, Higgins PJ (2011) A switch in RND3-RHOA signaling is critical for melanoma cell invasion following mutant-BRAF inhibition. Mol Cancer 10: 114 .

Klein RM, Spofford LS, Abel EV, Ortiz A, Aplin AE (2008) B-RAF regulation of Rnd3 participates in actin cytoskeletal and focal adhesion organization. Mol Biol Cell 19: 498-508.

Lessard L, Labbe DP, Deblois G, Begin LR, Hardy S, Mes-Masson AM, Saad F, Trotman LC, Giguere V, Tremblay ML (2012) PTP1B is an androgen receptor-regulated phosphatase that promotes the progression of prostate cancer. Cancer Res 72: 1529-1537.

Liang S, Sharma A, Peng HH, Robertson G, Dong C (2007) Targeting mutant (V600E) B-Raf in melanoma interrupts immunoediting of leukocyte functions and melanoma extravasation. Cancer Res 67: 5814-5820.
Ni CS, Sun BC, Dong XY, Sun T, Zhao N, Liu YR, Gu Q (2012) Promoting melanoma growth and metastasis by enhancing VEGF expression. Contemp Oncol (Pozn) 16: 526-531.

Park GB, Kim YS, Lee HK, Song H, Cho DH, Lee WJ, Hur DY (2010) Endoplasmic reticulum stress-mediated apoptosis of EBV-transformed B cells by cross-linking of $\mathrm{CD} 70$ is dependent upon generation of reactive oxygen species and activation of p38 MAPK and JNK pathway. J Immunol 185: 7274-7284.

Riou P, Villalonga P, Ridley AJ (2010) Rnd proteins: multifunctional regulators of the cytoskeleton and cell cycle progression. Bioessays 32: 986-992.

Ryan MC, Kostner H, Gordon KA, Duniho S, Sutherland MK, Yu C, Kim KM, Nesterova A, Anderson M, Mcearchern JA, Law CL, Smith LM (2010) Targeting pancreatic and ovarian carcinomas using the auristatin-based anti-CD70 antibody-drug conjugate SGN-75. Br J Cancer 103: 676-684.

Tilkin-Mariame AF, Cormary C, Ferro N, Sarrabayrouse G, Lajoie-Mazenc I, Faye JC, Favre G (2005) Geranylgeranyl transferase inhibition stimulates anti-melanoma immune response through MHC class I and costimulatory molecule expression. FASEB J 19: 1513-1515.

Vega FM, Ridley AJ (2008) Rho GTPases in cancer cell biology. FEBS Lett 582: 2093-2101.

Wischhusen J, Jung G, Radovanovic I, Beier C, Steinbach JP, Rimner A, Huang H, Schulz JB, Ohgaki H, Aguzzi A, Rammensee HG, Weller M (2002) Identification of CD70-mediated apoptosis of immune effector cells as a novel immune escape pathway of human glioblastoma. Cancer Res 62: 2592-2599.

This work is published under the standard license to publish agreement. After 12 months the work will become freely available and the license terms will switch to a Creative Commons AttributionNonCommercial-Share Alike 4.0 Unported License.

Supplementary Information accompanies this paper on British Journal of Cancer website (http://www.nature.com/bjc) 